\title{
Ulipristal acetate as a treatment option for uterine fibroids
}

\author{
Karolina Piecak, Paweł Milart, Ewa Woźniakowska, Tomasz Paszkowski \\ $3^{\text {rd }}$ Department of Gynecology, Medical University, Lublin, Poland
}

\begin{abstract}
Uterine fibroids are the most common benign uterine tumours. Clinical symptoms include abnormal bleeding, pelvic pressure, pelvic pain, infertility and obstetric complications. Approximately one third of women with fibroids will require treatment. The management also depends on the number, size, and location of the fibroids. There are surgical and non-surgical treatment options. The choice of therapy depends on different factors, such as the severity of symptoms, tumour characteristics, age, and wish to preserve the uterus and fertility. There is growing evidence of the main role of progesterone pathways in the pathophysiology of uterine fibroids due to the use of selective progesterone receptor modulators such as ulipristal acetate. The efficacy of long-term intermittent use of UPA was recently demonstrated by randomised controlled studies. There is great demand for alternatives to surgical intervention, especially in women seeking to preserve their fertility. One of these alternatives is ulipristal acetate, which is proven to treat fibroid symptoms effectively.
\end{abstract}

Key words: myomas, fibroids, selective progesterone receptor modulators, ulipristal acetate, pharmacological therapy.

\section{Introduction}

Uterine fibroids are the most common benign uterine tumours in women in the reproductive age group. They were first described in 1791 by Matthew Baillie of St. George's Hospital in London. They are also known as myomas or leiomyomas and occur in $20-50 \%$ of women. Symptoms may vary and include dysmenorrhoea, heavy and prolonged menstrual bleeding, anaemia, pelvic pain and pressure, infertility, and recurrent miscarriages and may result in reduction of quality of life. Many different management strategies are used, such as surgical treatment (myomectomy/hysterectomy by laparoscopy/ laparotomy/hysteroscopy, uterine artery embolization), nonsurgical procedures (uterine artery embolisation, MRI-guided focused ultrasound) as well as medical therapy. The choice of the appropriate treatment technique is made on the basis of many factors such as fibroid location, patient's age, the wish to preserve fertility, concomitant diseases, and the patient's preferences. Hysterectomy remains the most common treatment option because it is the only definitive treatment and it eliminates the possibility of recurrence [1, 2].

Medical therapy with progestins, progestin-releasing intrauterine devices, and gonadotropin-releasing hormone agonists ( $\mathrm{GnRHa}$ ) are also approved.

Pharmacological treatments such as gonadotropinreleasing hormone agonists and antagonists have been used with only partial success. Countless side effects (including bone loss and hot flushes) and limited clinical effect decreased their role and exposed a need for new effective medical treatments. Treatment with selective progesterone receptor modulators has shown promising results with shrinkage of uterine leiomyomas and a prolonged clinical effect $[1,2]$.

\section{Mode of action}

After proving the crucial role of progesterone in the growth and development of uterine leiomyomas, it has been shown that selective progesterone receptor modulators (SPRMs) might offer another potent therapeutic option to treat uterine leiomyomas.

Progesterone plays a role in promoting the growth of fibroids. This fact opens the possibility of using selective progesterone receptor modulators (SPRMs), while the effects of progesterone on target tissues are modified by progesterone receptors. SPRMs are synthetic compounds that compete at the progesterone receptor binding site, demonstrating either agonist or antagonist activity on progesterone receptor [1, 2].

Whether SPRMs act predominantly as antagonists or agonists depends on their structure and how it changes the progesterone receptor conformation, leading to exposure or inactivation of particular binding domains, which leads to interactions of corepressors and/ or coactivators with the progesterone receptor. 

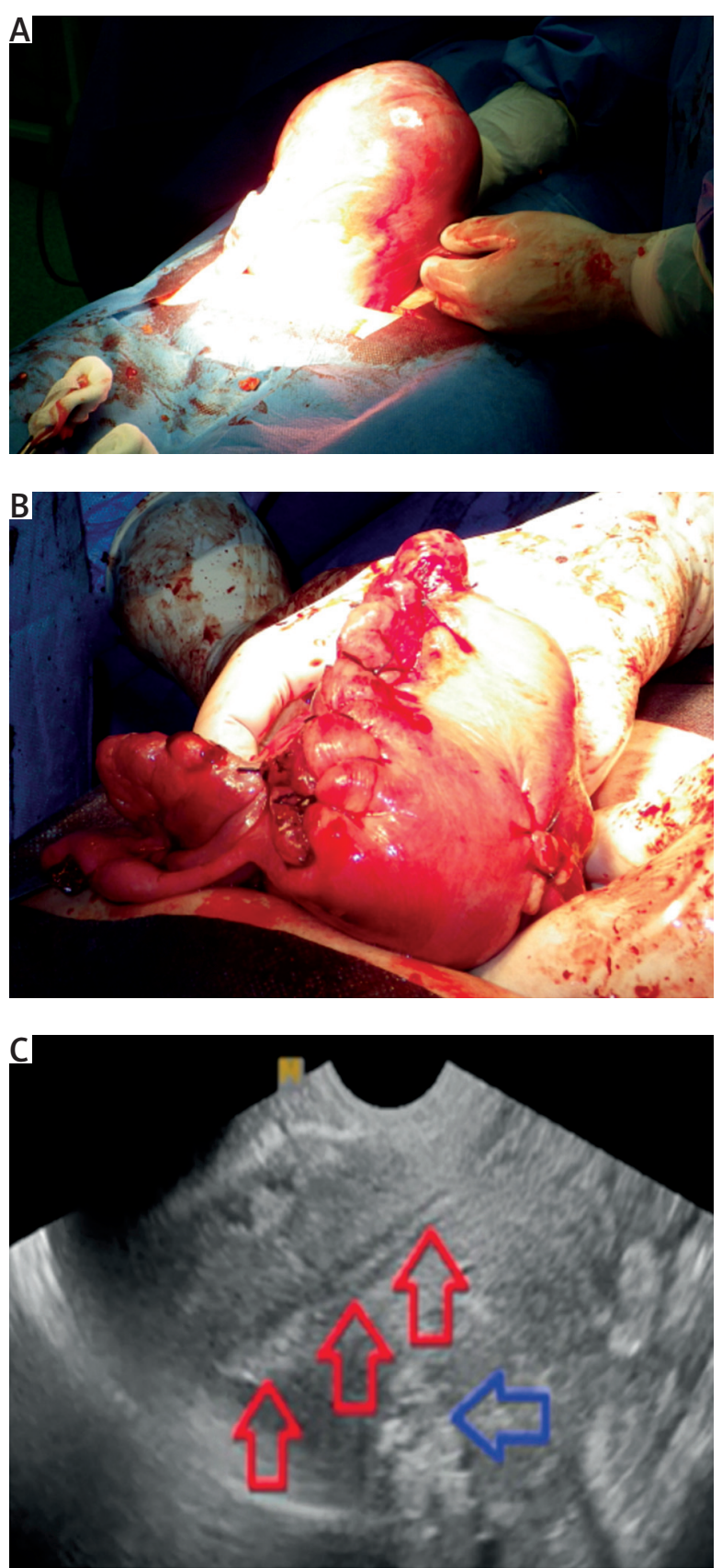

Fig. 1. A) Surgical view of a large fibroid that decreased its volume by $40 \%$ after preoperative ulipristal acetate treatment. B) Because of the patient's wish to become pregnant a myomectomy was performed. C) Ultrasound performed 3 months after the procedure showed a normal uterine cavity (red arrows) and a normal post-myomectomy scar in the posterior uterine wall (blue arrow). The patient conceived 12 months later

In-vitro studies have shown that progesterone stimulates the proliferation of fibroid cells. These studies also demonstrated that SPRMs (asoprisnil, telapristone acetate, and UPA) inhibit cell proliferation. This process induces apoptosis selectively in fibroid cells by downregulating antiapoptotic factors, antifibrotic activity, and reducing or blocking growth factor expression.
Different mechanisms of action of SPRMs may contribute to their efficacy in decreasing the size of fibroids and reducing the heavy blood loss associated with their presence, such as a direct effect on uterine blood vessels.

SPRMs have a particular effect on endometrium tissue. The mechanism is described as PRM-associated endometrial changes (PAECs) and recognised by a cystic dilatation of endometrial glands, an irregular architecture lined with inactive gland cells, and a nondecidualised stroma and does not occur in physiological states. The endometrium could not be classified as proliferative or secretory. PAECs are characterised by an inactive proliferating epithelium, associated with asymmetry of stromal and epithelial growth with mixed mitotic and secretory epithelial effects [1, 2].

\section{Use of ulipristal acetate for preoperative management of uterine fibroids: results from the PEARL I and PEARL II clinical trials}

The PEARL (PGL4001 Efficacy Assessment in Reduction of symptoms because of uterine Leiomyomata) I and PEARL II trials were published in 2012 when 5 mg UPA obtained European approval to be used for threemonth preoperative treatment of women with uterine fibroids.

PEARL I was a randomised, double-blind, placebocontrolled trial evaluating three-month treatment with UPA (tablets with 5 or 10 mg UPA daily) in women with symptomatic fibroids, heavy uterine bleeding, and anaemia. The study results have shown that after 13 weeks, uterine bleeding was under control in $91 \%$ of women receiving $5 \mathrm{mg}$ UPA, $92 \%$ of women receiving $10 \mathrm{mg}$ UPA, but only in $19 \%$ of women receiving a placebo. Most patients achieved improvement of anaemia (haemoglobin $>12 \mathrm{~g} / \mathrm{dl}$ ) by the end of the three-month treatment period with UPA. It has been well documented that preoperative anaemia, even in a mild degree, is associated with increased risk of morbidity and mortality in patients undergoing surgery.

PEARL II compared 5 and 10 mg UPA with the standard medical treatment with $\mathrm{GnRH}$ (3.75 mg leuprolide acetate depot injection once monthly).

The findings of this study demonstrated that uterine bleeding was controlled in $90 \%$ of patients receiving $5 \mathrm{mg}$ UPA, $98 \%$ of patients receiving $10 \mathrm{mg}$ UPA, and $89 \%$ of patients receiving leuprolide acetate. The median times to controlled bleeding were five to seven days for patients receiving UPA (5 and $10 \mathrm{mg}$, respectively), and 21 days for patients receiving leuprolide acetate.

Follow-up in a group of women who did not undergo surgery after the three-month UPA treatment showed that UPA had a sustained effect (up to six months) after the end of treatment [1-3]. 
Long-term medical management of uterine fibroids: results of PEARL III and PEARL IV clinical trials

The PEARL III study was designed to estimate the efficacy and safety of long-term intermittent open-label three-month courses of $10 \mathrm{mg} /$ day UPA for the treatment of symptomatic uterine fibroids. After the first UPA course, amenorrhoea occurred in $79 \%$ of women. Amenorrhoea rates were, respectively, 89, 88, and 90\% in women who received two, three, and four treatment courses. The median time to amenorrhoea was 3.5 days from the start of treatment.

The median fibroid volume reduction from the baseline was 49.9, 63.2, 67.0, and 72.1\% after one, two, three, and four courses of treatment, respectively.

All endometrial biopsies showed benign histology without hyperplasia.

PEARL III has demonstrated that administration of more than one course of UPA maximises the potential benefits of treatment when it comes to bleeding control and fibroid volume reduction.

PEARL IV evaluated the efficacy and safety of repeated 12-week courses of 5 or 10 mg UPA daily for intermittent treatment of symptomatic uterine fibroids. After the second treatment course, the median reduction in fibroid volume from the baseline was 54 and $58 \%$ in patients receiving 5 and $10 \mathrm{mg}$ UPA, respectively. Improvement of pain and quality of life has been observed.

Pregnancies after UPA treatment have been reported. Twenty-one women wanted to conceive - obtaining 18 pregnancies, which resulted in 12 births of 13 healthy babies and six early miscarriages [1-3].

\section{Long-term intermittent use of $10 \mathrm{mg}$ UPA in repeated courses of three months}

The efficacy of long-term intermittent use of $10 \mathrm{mg}$ UPA given in repeated courses of three months, each course separated by two menstrual cycles was analysed. The long-term intermittent therapy maximises the efficacy of UPA. Control of bleeding is achieved sooner after each course and the time to amenorrhoea is shorter. With each subsequent course, a significantly greater number of patients achieved a fibroid volume reduction of more than $50 \%$.

Slight differences exist in the algorithms of UPA treatment concerning the age, the symptoms, and the demands of the patients. The choice of fibroid therapy should be based on factors such as: the severity of symptoms (pain, bleeding), infertility connected to fibroids, fibroid characteristic (localisation, volume), age, wish to preserve the uterus, and wish to preserve fertility $[1,4-6]$.
Donnez et al. proposed novel treatment algorithms:

Women < 40 years of age presenting with symptomatic myomas deforming the uterine cavity and infertility.

If type 0 myomas (International Federation of Gynaecology and Obstetrics classification) are present, cutting the pedicle by hysteroscopy is indicated and no preoperative treatment is required.

For type 1 myomas $(<3 \mathrm{~cm})$, two options are recommended: hysteroscopic myomectomy or UPA treatment in one or two courses of three months, followed by hysteroscopic myomectomy in the case of an inadequate response. In most cases, hysteroscopic myomectomy for type 1 myomas is relatively easy to perform.

For type 1 myomas (>3 cm) or type 2 , UPA may be given in one or two courses of three months. Type 1 and 2 myomas often respond to this preoperative therapy and regress in size. The reduction allows a hysteroscopic myomectomy that can be planned after the first menstrual bleed induced by NETA (10 mg/10 days), administered immediately after UPA treatment. In some cases (if myomas regress so much that they no longer distort the uterine cavity), surgery may not be required.

If the myomas are multiple (two to six) or of different classes (class 2-5), UPA can be given in two courses of three months.

After these two courses of three months, there are four possible outcomes:

1. Myoma regression is very significant (> 50\% decrease in volume). The uterine cavity is not distorted, and the patient can try to conceive naturally or undergo assisted reproductive techniques.

2. Myoma regression is significant (> $25 \%$ but $<50 \%$ ), but the baseline volume is so great that the indication for surgery remains. In this case, UPA may allow surgery to be performed by a laparoscopic approach (when the haemoglobin level is normalised), avoiding laparotomy.

3. Myoma regression is moderate (> $25 \%$ but $<50 \%$ ), but the uterine cavity remains distorted. In this situation, two options should be considered with the patient: prolong medical therapy for another two courses of three months or offer surgical treatment.

4. Myoma regression is insufficient. Surgical indications remain according to the size and localisation of myomas $[1,6,7]$.

Premenopausal women presenting with symptomatic myomas but no desire for pregnancy, who wish to preserve their uterus.

If type 0 or type 1 myomas are present, the same algorithm as among the women < 40 years of age with symptomatic myomas deforming the uterine cavity can be applied. 
In the case of type 2 fibroids distorting the uterine cavity or multiple large myomas (class 2-5), long-term intermittent therapy may be proposed. If, after two courses of three months, myoma regression (>50\%) is satisfactory and the patient is free of symptoms, she can be observed until symptoms recur. Additional courses of UPA may then be proposed. No data are so far available to determine the interval before recurrence. Donnez et al. do not state that UPA will eradicate all indications for myomectomy, but instead that surgery can be avoided in the case of a very good response, or at least be postponed until the patient wishes to become pregnant. In the case of insufficient fibroid volume reduction or persistent bleeding, myomectomy is still indicated $[1,6,7]$.

Women $>40$ years of age presenting with symptomatic myomas.

In premenopausal women with symptomatic myomas, long-term intermittent therapy may be proposed. In the case of moderate response ( $>25 \%$ but $<50 \%$ reduction), two additional UPA courses can be proposed.

In the case of insufficient response in terms of fibroid volume reduction but good control of bleeding, it may be possible to wait until bleeding recurs. In this case, UPA treatment or performing hysterectomy or uterine artery embolisation may be available options. In the case of persistent heavy bleeding, surgery is indicated $[1,6-8]$.

\section{Conclusions}

In conclusion, UPA is the most effective pharmacological management of fibroids and in many cases it may be an alternative to surgical treatment. This review shows the possible place of UPA as a preoperative option or as a medical therapy alternative to surgery, the safety profile, and prolonged effects with improvement of symptoms, quality of life, and pain even after cessation of the therapy.

\section{Disclosure}

Authors report no conflict of interest.

\section{References}

1. Donnez J, Arriagada P, Donnez O, Dolmans MM; Current management of myomas: the place of medical therapy with the advent of selective progesterone receptor modulators. Curr Opin Obstet Gynecol 2015; 27: 422-431.

2. Safrai M, Chill H, Salzman R, Shushan A; Selective progesterone receptor modulators for the treatment of uterine leiomyomas. Obstet Gynecol 2017; 130: 315-318.

3. Donnez J, Donnez O, Dolmans MM. Safety of treatment of uterine fibroids with the selective progesterone receptor modulator, ulipristal acetate. Expert Opin Drug Saf 2016; DOI:10.1080/14740338.2016.1248943

4. Donnez J, Dolmans MM. Uterine fibroid management: from the present to the future. Hum Reprod Update 2016; 22: 665-686.
5. Murji A, Whitaker L, Chow TL, Sobel ML. Selective progesterone receptor modulators (SPRMs) for uterine fibroids. Cochrane Db Syst Rev 2017; 4: CD010770

6. Donnez J, Hudecek R, Donnez O, et al. Efficacy and safety of repeated use of ulipristal acetate in uterine fibroids. Fertil Sertil 2015; 103: 519-27.e3

7. Kalampokas T, Kamath M; Ulipristal acetate for uterine fibroids: a systematic review and meta-analysis. Gynecol Endocrinol 2016; 32: 91-99.

8. Pérez-López FR; Ulipristal acetate in the management of symptomatic uterine fibroids: facts and pending issues. Climacteric 2015; 18: 177-181. 